\title{
MEDICAL ERROR, PERSISTENT VEGETATIVE STATE AND OVER NINETEEN LONG YEARS OF MEDICAL CARE
}

\author{
Zahid Farooq Baig, Aslam Khan, Ibrar Hussain Zaidi, Muhammad Azmat Khan, Muhammad Salman Ashraf \\ Combined Military Hospital Peshawar/National University of Medical Sciences (NUMS) Pakistan
}

\begin{abstract}
A medical error is a preventable adverse effect of medical care. Errors can happen in the hospital at the doctor's office, at the pharmacy, laboratory and ward. These can also occur at home by the patient or the attendant. These errors may lead to death or major/minor injuries or can also be harmless. However these should be recorded, analysed and remedial measures implemented to reduce the risk of being committed in the future. We are reporting the medical care of our patient in persistent vegetative state over nineteen years due to medical error at our hospital resulting in brain anoxia. The key issues faced during the care were recurrent respiratory and urinary tract infections and in last one year kidney calculi leading to chronic kidney disease and its complications. There are no contractures or thrombo-embolic events and above all no bed sore over this period.
\end{abstract}

Keywords: Chronic kidney disease, Long term care, Medical error, Persistent vegetative state.

This is an Open Access article distributed under the terms of the Creative Commons Attribution License (https://creativecommons.org/licenses/by-nc/4.0/), which permits unrestricted use, distribution, and reproduction in any medium, provided the original work is properly cited.

\section{INTRODUCTION}

We learn from our mistakes. History of medical errors could be traced back to the start of medical care. Medical errors are still a common cause of mortality and morbidity in the patients. A study by researchers at Johns Hopkins Medicine reveals that medical errors should rank the third leading cause of death in United States, and continue to be an under-recognised cause of death ${ }^{1}$. In Pakistan, due to the low medical staff to patient ratio, less developed medical facilities and a weak culture of recording such cases, the frequency of medical errors may be very high.

Medical errors range from mistakes by doctors, paramedics, pharmacists or the patient. These can happen at the hospital in doctor's clinic, ward, laboratory, radiology or at patients home. These errors can be harmless or can lead to disabilities like persistent vegetative state as in our case or even death.

The term "persistent vegetative state" (PVS) was coined by Jennett et al in 1972 to describe the condition of patients with severe brain damage in whom coma has progressed to a state of wakefulness without detectable awareness ${ }^{2}$. PVS is judged to be permanent after three months if induced non-traumatically and one year for traumatic brain injury.

\section{CASE REPORT}

It was $10^{\text {th }}$ of November 1999 when my patient, a young pregnant lady 20 years of age, was admitted to Combined Military Hospital (CMH) Peshawar with

Correspondence: Dr Zahid Farooq Baig, Department of Nephrologist, Combined Military Hospital, Multan Pakistan

Received: 04 Jan 2018; revised received: 02 Feb 2021; accepted: 09 Feb 2021 oligohydramnios. Due to expected preterm birth, dexamethasone (Decadron) was prescribed by gynaecologist. The first dose of dexamethasone was administered at 1600 hours and the next dose was due at 2400 hours. The second dose which was issued by pharmacist was "Pavulon (Pancuronium bromide)" instead of "Decadron." Pavulon is used by anaesthetists as a neuromuscular blocker during general anaesthesia. The gynaecologist had advised injection "Decadron" to two other patients as well on that date and all three were issued "Pavulon." Unfortunately my patient was the first lady to whom it was administered. Duty nurse noticed a different drug, inquired from medical store about this but was told that it is the same drug with a different trade name. After administration the patient immediately went into respiratory arrest. She started gasping became cyanosed and there was a generalized twitching over the body. This was noticed by her attendant who called the nurse. Call was sent to the gynaecologist and medical specialist and patient shifted to operation theatre (OT). Cardio-pulmonary resuscitation was started and return of spontaneous circulation achieved after 5 minutes of reaching OT. Endo-tracheal (ET) tube was passed, a crash caesarean section performed and baby girl was delivered. Post operatively the patient was shifted to intensive care unit (ITC), clinically her pupils were fixed and dilated and she had extensor response to painful stimuli. As the sedation was decreased, she became irritable and started having recurrent fits. Slowly the pupils became responsive and fits were controlled by anti-epileptic medications but her irritability persisted. She was weaned off mechanical ventilation on 19th Nov 1999 (day 9), but remained on ET tube. Tracheostomy was carried out on $3^{\text {rd }}$ 
Dec 1999 (day 23). CT scan of brain was suggestive of ischemic brain injury. Echocardiography was within normal limits. During this immediate post operative period she developed other complications like septicaemia, tube blockade, and urethral injury with hematuria secondary to Foley catheterization and catheter leak etc. Her irritability settled by 10 weeks. For feeding purpose naso-gastric (NG) tube was passed as family was not willing for percutaneous endoscopic gastrostomy (PEG).

A diagnosis of PVS secondary to brain anoxia was made. Then the story of palliative medical care started which continued for over nineteen years of the initial event. Since then she is admitted in $\mathrm{CMH}$ Peshawar remained in main ITC for ten years and then shifted to a side room in high dependency unit (HDU). During this period many hospital administration, medical, surgical and physiotherapy teams have changed but her palliative care was continued. Her family was always there providing excellent social support and she was considered to be the most important patient of the hospital. She was provided nutrition through NG tube and she remained on Foley catheter for eight years and after that she remained on diapers, changed regularly on required basis. Tracheostomy was removed after 13 years. During this time she had multiple complications including urinary tract and respiratory tract infections, conjunctivitis, episodes of vomiting, problems with NG tube, tracheostomy and Foley catheter. A board of creatinine level and a fall in haemoglobin. Platelet count more or less remained constant. Table-I shows the mean level of haemoglobin, platelets, urea and creatinine between years 2011 and 2017.

Her blood pressure remained within limits mostly in low normal range till mid 2015 and then started to rise and in 2016 she is on Amlodipine 10mg daily to control the blood pressure. I remained her treating physician for 2 years from 2015-2017. At that time she had mild pallor with stable vital signs and had a normal sleep and wake up cycle. When awake she was not aware about self and environment and did not interact. There were no purposeful response to any type of stimuli and she had no control over her bowel and bladder. There were occasional episodes of weeping. She had clenched fists posture and there was increased tone in all limbs with exaggerated reflexes. Pupillary light reflex, corneal and gag reflexes were intact. Examination of cardiovascular, respiratory and gastrointestinal systems was within normal limits.

During the course of the illness, my patient developed nephrolithiasis leading to chronic kidney disease and hypertension. In 2017 her creatinine level was 215 umol/L, with an estimated glomerular filtration rate (GFR) of $30 \mathrm{ml} / \mathrm{min}$. Ultrasound revealed small right kidney and hydronephrotic left kidney. Urologist had advised against any active intervention for this. She was anaemic with the haemoglobin of $11 \mathrm{gm} / \mathrm{dl}$ and was on haematinics and erythropoietin for this. Echo-

Table-I: Mean laboratory values from 2011 to 2017.

\begin{tabular}{c|c|c|c|c}
\hline Year & Hemoglobin $\mathbf{( g m / d l )}$ & Platelets (x109/L) & Urea (mmol/L) & Creatinine (umol/L) \\
\hline 2011 & $12.5 \pm 1.21$ & $310 \pm 52.18$ & $4.6 \pm 1.56$ & $105 \pm 24.75$ \\
\hline 2012 & $11.3 \pm 2.04$ & $328 \pm 39.34$ & $4.5 \pm 1.98$ & $114 \pm 54.82$ \\
\hline 2013 & $13.5 \pm 1.61$ & $223 \pm 90.42$ & $6.4 \pm 3.24$ & $125 \pm 68.24$ \\
\hline 2014 & $12.3 \pm 2.27$ & $317 \pm 66.91$ & $7.1 \pm 2.86$ & $135 \pm 36.40$ \\
\hline 2015 & $11.8 \pm 1.62$ & $306 \pm 45.82$ & $9.1 \pm 2.54$ & $150 \pm 48.23$ \\
\hline 2016 & $10.7 \pm 1.37$ & $331 \pm 58.86$ & $8.8 \pm 4.92$ & $190 \pm 141.44$ \\
\hline 2017 & $11.0 \pm 2.09$ & $332 \pm 68.12$ & $9.5 \pm 3.66$ & $215 \pm 116.69$ \\
\hline
\end{tabular}

doctors used to review the patient regularly and adjust treatment and do family counselling about her latest condition.

The most commendable thing for the hospital and family of this patient is the fact that during these over nineteen years of care there has not been a single episode of bed sore. This fact speaks out about the level of care this patient had received.

After initial stabilization of the patient, the laboratory values remained within normal limits till 2011 after which there is a tendency of rising urea and cardiography revealed left ventricular hypertrophy with ejection fraction of $30-35 \%$. Being on NG feeding tube for such a long time, she developed frequent episodes of sinusitis and pneumonitis for which she has been given multiple courses of antibiotics. Finally, on $2^{\text {nd }}$ June 2017 PEG tube was passed and with this her nutrition improved, her weight which has remained constant between 40-45 kilograms increased to 65 kilograms.

Her medications included oral haematinics, Tab Sevelamer as phosphate binder, Alphacalcidol, Tab 
Amlodipine, Inj Erythropoietin, Tab Pentroprazole, Tab Baclofen (to decrease spasticity) and Tab Levetiracetam (for fits).

Coming to final words of the event, an inquiry was ordered to find out the facts and those at fault were punished. The baby girl could only survive for 8 days. The other 2 injections which were issued to other patients were fortunately withdrawn after the incident.

Patient remained under care of multiple disciplinary team with regular trouble shooting of the complications she faced. On 23 ${ }^{\text {rd }}$ April 2019 she had a severe episode of gastro-intestinal bleed and could not be reviewed despite all possible efforts and left for her heavenly abode. She lived for 19 years, 5 months and 13 days speaking high of the level of palliative care provided by the medical team after her initial event.

\section{DISCUSSION}

The PVS occurs as the result of severe brain damage. Recovery from a non-traumatic PVS after three months is exceedingly rare in both adults and children. The life span of adults and children in such a state is substantially reduced. For most such patients, life expectancy ranges from two to five years; survival beyond ten years is unusual ${ }^{3}$.

In long term care of our patient we were able to prevent many complications which are expected in patients with prolonged bed rest and immobilization. Almost every organ can be affected with prolonged immobilization 5,6 . The main problems which we faced in our patient include kidney stones leading to CKD and its complications and recurrent respiratory and urinary tract infections.

The likely sequence of events for CKD in our patient includes osteoporosis leading to bone resorption and kidney stones formation and thus causing obstructive nephropathy and chronic pyelonephritis. Decreased muscle activity and decreased weight bearing in immobilized patients leads to changes in calcium homeostasis, resulting in resorption of skeletal bones leading to hypercalciuria, hyperphosphaturia and in some patients hypercalcemia. This may lead to kidney stone formation, with affected patients usually forming calcium phosphate stones ${ }^{7}$. There is a consistent relationship between history of kidney stones and an increased risk of $\mathrm{CKD}^{8}$.

The presence of hypertension, left ventricular hypertrophy $(\mathrm{LVH})$, anaemia and low ejection fraction can all be part of spectrum of complications of CKD. Hypertension is present in approximately $80-85 \%$ of patients with $\mathrm{CKD}^{9}$. The prevalence of hypertension rises as the glomerular filtration rate falls ${ }^{10}$. LVH has been found in as many as 47 percent of patients with CKD, not yet on dialysis. There is higher prevalence and more severe LVH in those with increasingly lower degree of kidney function. The risk factors for development of LVH in patients with CKD include anaemia, and hypertension.

The palliative course of our patient included episodes of respiratory tract and urinary tract infections. Urinary tract infection is the most frequent bacterial infection in residents of long-term-care facilities. Most infections are asymptomatic, with a remarkable prevalence of asymptomatic bacteriuria of $15-50 \%$ among all residents. In the non-catheterized resident, urinary infection is an infrequent source of fever but may not be definitively excluded. The presence of NG tube predisposes to nasal and maxillary sinus inflammation.

\section{ACKNOWLEDGEMENT}

We will like to appreciate the efforts of all the doctors and paramedical staff who directly or indirectly remained involved in the care of this patient and also to the family of the patient who were always there for the support.

\section{CONCLUSION}

In a country like Pakistan where long term care facilities are almost nonexistent, palliative care is a big challenge in already under-staffed hospitals. It is a multi-disciplinary team work, requiring high level of commitment and becomes more effective with a good family support. Over nineteen years of care with no bed sores, contractures, thrombo-embolic events speak of very high quality of care.

\section{CONFLICT OF INTEREST}

This study has no conflict of interest to be declared by any author.

\section{REFERENCES}

1. Makary MA, Daniel M. Medical error-the third leading cause of death in the US. Br Med J 2016; 353(2): i2139-42.

2. Jennett B, Plum F. Persistent vegetative state after brain damage: a syndrome in search of a name. Lancet 1972; 1(1): 734-37.

3. Multi-society task force on PVS. Medical aspects of the persistent vegetative state. N Engl J Med 1994; 330(30): 1499-1508.

4. ACOG Committee on Obstetric Practice. ACOG Committee Opinion no 475. Antenatal corticosteroid therapy for fetal maturation. Obstet Gynecol 2011; 117(2 pt 1): 422-24.

5. Rainfray M, Dehail P. Complications of immobility and bed rest. Prevention and management. Rev Prat 2007; 57(6): 671-76.

6. Maniere D. Complications of immobility and bed rest. Prevention and management. Rev Prat 2012; 62(7): 1013-23.

7. Okada A, Ohshima H, Itoh Y, Yasui T, Tozawa K, Kohri K. Risk of renal stone formation induced by long-term bed rest could be 
decreased by premedication with bisphosphonate and increased by resistive exercise. Int J Orol 2008; 15(7): 630-35.

8. Keddis MT, Rule AD. Nephrolithiasis and loss of kidney function. Curr Opin Nephrol Hypertens 2013; 22(4): 390-96.

9. Whaley-Connell AT, Sowers JR, Stevens LA, McFarlane SI, Shlipak MG, Norris KC, et al. CKD in the United States; kidney early evaluation program (KEEP) and national health and nutrition examination survey (NHANES) 1999-2004. Am J kidney Dis 2008; 51(2): S13-20.

10. Velasquez MT, Beddhu S, Nobakht E, Rahman M, Raj DS. Ambulatory blood pressure in chronic kidney disease: ready for prime time. Kidney Int Rep 2016; 1(2): 94-104. 